\title{
In Vivo and In Vitro Studies on Virulence and Host Responses to Saccharomyces cerevisiae Clinical and Non-Clinical Isolates
}

\author{
Alberto Yáñez ${ }^{1}$, Celia Murciano ${ }^{1}$, Silvia Llopis ${ }^{2}$, Teresa Fernández-Espinar ${ }^{2}$, M. Luisa Gil ${ }^{1}$ and \\ Daniel Gozalbo ${ }^{*}, 1$
}

\author{
${ }^{I}$ Departamento de Microbiología y Ecología, Universitat de València, Avda. Vicent Andrés Estellés s/n, E-46100 \\ Burjassot, Spain \\ ${ }^{2}$ Departamento de Biotecnología, Instituto de Agroquímica y Tecnología de Alimentos (CSIC), P.O. Box 73, E-46100 \\ Burjassot, Spain
}

\begin{abstract}
We have studied the virulence and host responses to several clinical and non-clinical Saccharomyces cerevisiae isolates: two vaginal isolates $(60,61)$, one isolate from faeces (20), a brewer's yeast isolate used in dietetics (D14), one $S$. boulardii isolate from a commercial probiotic product, and a reference natural wine yeast (CECT 10431). Hematogenously disseminated infection in a mouse model demonstrated that four isolates (all, except 20 and 10431) were able to colonize preferentially the brain, as well as kidney and spleen, to a lesser extent, of immunocompetent mice. In vitro adhesion assays to epithelial and endothelial cell lines also showed an increased adherence ability of strains 60, 61, D14 and S. boulardii. In vitro cytokine production assays by RAW 264.7 murine macrophages challenged with yeasts showed a relative increased production of TNF- $\alpha$ in response to the 20 and 10431 strains; viability of RAW cells after coculture was similar in all cases (2-5\% non-viable cells) except for 60 strain (11\% non-viable cells). In vitro phagocytosis assays of yeasts by RAW cells showed that two isolates (D14 and particularly S. boulardii) were engulfed less efficiently. These results point out that $S$. cerevisiae isolates, from both clinical and non-clinical (dietetic and probiotic) origin, may vary in the expression of putative virulence factors contributing to their ability to develop the infectious process.
\end{abstract}

Keywords: Saccharomyces cerevisiae, virulence, adhesion, proinflammatory cytokines, phagocytosis.

\section{INTRODUCTION}

Saccharomyces cerevisiae strains are the most widely used yeast in industrial food and beverage production, traditionally regarded as absolutely safe, and even $S$. boulardii, considered as a subtype of $S$. cerevisiae, has been described as a biotherapeutic agent in the prevention and treatment of antibiotic-associated diarrhoea and colitis in humans [1-3]. Moreover, S. cerevisiae strains can colonize the respiratory, gastrointestinal and genitourinary tracts in a saprophytic way without causing disease; however, in the last decades there is increasing evidence indicating that $S$. cerevisiae is responsible for superficial diseases, that are not life-threatening, although in severely immunocompromised individuals serious systemic infection may occur [4-7]. Improvement of the identification at the species level, the increasing population of immunocompromised individuals, the widespread use of immunosuppressive therapies and broad-spectrum antibacterial chemotherapy, as well as the use of permanent intravascular catheters are factors contributing to this phenomenon [7]. Most nosocomial cases of $S$. cerevisiae fungemia in immunocompromised or critically-ill patients appear to arise from the use of $S$. boulardii as a biotherapeutic agent, although baker's yeasts

*Address correspondence to this author at the Departamento de Microbiología y Ecología, Facultad de Farmacia, Universitat de València, Avda. Vicent Andrés Estellés s/n, 46100 Burjassot, Spain; Tel: (+34) 96354 3026; Fax: (+34) 96354 4543; E-mail: daniel.gozalbo@uv.es have been also isolated from blood [8-10]. The main routes for bloodstream infections are enteral translocation of ingested yeasts and direct contamination of the central venous catheter insertion site [11-14]. In addition, severe infections by $S$. cerevisiae have been occasionally reported in patients with no obvious predisposing factors $[15,16]$. Using molecular techniques to characterize $S$. cerevisiae clinical isolates, both $S$. boulardii and baker's yeasts were identified [10, 17]; the link between a subset of clinical isolates and probiotic or baking S. cerevisiae strains has been confirmed by phylogenetics and metabolomics [18]. The association between clinical isolates and non-clinical strains points out to food and probiotic/biotherapeutic strains from Saccharomyces species as a possible origin of human systemic infections. Therefore, as the reports of $S$. cerevisiae infections are increasing steadily, this yeast species is now considered as an emerging low virulence opportunistic pathogen rather than non-pathogenic yeast [4-7].

Similarly to other opportunistic fungal pathogens, such as its close relative Candida species, the ability to colonize and cause disease in the host may depend on a delicate balance between the immune status of the host and the expression of microorganism-related putative virulence factors $[19,20]$. These factors are still poorly understood, as the interest in $S$. cerevisiae as an opportunistic pathogen is recent and there are few studies about the origin and mechanisms of infection [21-23]. These reports describe physiological traits with no direct evidence of their role during infection; the majority of 
the clinical isolates secrete higher levels of protease and phospholipase, grow better at $42{ }^{\circ} \mathrm{C}$, and show strong pseudohyphal growth relative to industrial yeasts. Similarly, the information concerning the interactions between $S$. cerevisiae and the host defences is scant, and murine models have been studied with varying results [22, 24-29]. Comparison of $S$. cerevisiae clinical and non-clinical strains by molecular typing and determination of putative virulence traits has revealed that no specific virulence factor clearly separates the strains of clinical origin from the strains of non-clinical origin [30]. Adhesion to host tissues and recognition of fungal ligands by pattern recognition receptors (PRRs) that trigger innate immune responses (such as phagocytosis and cytokine production) are key mechanisms for initiating colonization and determining the fate of the infectious process $[19,20,31]$. It is well known in pathogenic fungi that the cell wall, as the outermost structure of the cell, plays a major role in the interactions with the host [32]. In this context, the $S$. cerevisiae-derived cell wall particle Zymosan has been widely used to study host responses such as phagocytosis and cytokine production, through recognition by PRRs [33-37].

In this work we have studied the virulence in a mouse model and the in vitro host responses to different clinical and non-clinical $S$. cerevisiae isolates (two vaginal isolates, one isolate from faeces, a brewer's yeast isolate used in dietetics, one $S$. boulardii isolate used as a biotherapeutic agent, and a reference wine strain) in order to define yeast traits related to virulence and their association with clinical and/or nonclinical isolates.

\section{MATERIALS AND METHODOLOGY}

Yeast Strains and Culture Conditions. Six $S$. cerevisiae strains (three clinical and three non-clinical) were used in this study (Table 1): two closely related commensal isolates from vagina (strains 60 and 61), and a commensal isolate from faeces (strain 20), collected by the Microbiology Service of the Vall d'Hebron Hospital (Barcelona, Spain) and characterized by molecular techniques [17]; a brewer's yeast isolate used in dietetics ( $S$. cerevisiae D14 strain), obtained from a commercial dietetic product at the Instituto de Agroquímica y Tecnología de Alimentos (CSIC, Burjassot, Spain); a strain of $S$. cerevisiae var boulardii ( $S$. boulardii) isolated from a commercial probiotic preparation [17], and the reference CECT (Spanish Type Culture Collection) 10431, a natural wine strain [23]. All selected yeast strains, except $S$. cerevisiae CECT 10431, exhibit potential virulence factors such as growth at $42{ }^{\circ} \mathrm{C}$, secretion of protease and phospholipase activities and pseudohyphal growth [23] (Table 1). Also two C. albicans strains were used in some assays: the high virulence ATCC 26555 strain and the PCA2 strain, a non-germinative lowvirulence strain, widely used in host-fungus interaction studies [38-41]. Yeast strains were routinely grown on YPD ( $1 \%$ yeast extract, $2 \%$ peptone, $2 \%$ glucose) plates cultured at $28{ }^{\circ} \mathrm{C}$ for $24 \mathrm{~h}$. Exponentially growing cultures were performed in liquid YPD at $28^{\circ} \mathrm{C}$ for $16-20 \mathrm{~h}$.

Endotoxin-free starved viable and fixed (inactivated) yeasts were obtained following standard procedures as previously described $[42,43]$. All the assays were performed under conditions to minimize endotoxin contamination. Endotoxin-free water and phosphate buffered saline (PBS) were used; liquid culture media were passed through a endotoxin removing gel (Pierce, Rockford, IL, USA) and tested for the absence of endotoxin by the E-toxate assay (Sigma-Aldrich, Madrid, Spain).

Infection Model in Mouse. Female mice (8-10 weeks old) of three different inbred strains (DBA/2, C3H/HeN and C57BL/6) were used (Harlan Ibérica, Barcelona, Spain). All assays involving mice were approved by the Institutional Animal Care and Use Committee.

Starved viable yeasts were diluted in endotoxin-free PBS to the appropriate cell concentration. Mice $(n=14)$ were challenged intravenously (i.v.) with $2 \times 10^{7}$ yeast cells (as determined by microscopic counting) in a volume of $0.2 \mathrm{ml}$ PBS, and survival was checked daily for three weeks. To assess the tissue outgrowth of the microorganism, at the indicated days post-infection the colony-forming units (cfu) were determined in homogenized organs (spleen, kidney and brain) from three infected animals following standard procedures.

Cell Lines, Growth Conditions and Adhesion Assays. The human umbilical vein endothelial cell line ECV304 (ECACC-92091712) was grown in M-199 cell culture medium supplemented with $10 \%$ heat-inactivated fetal bovine serum (FBS), $2 \mathrm{mM}$ L-glutamine, and $1 \%$ penicillin/streptomycin stock solution (Gibco, Barcelona, Spain), at $37^{\circ} \mathrm{C}$ in $5 \% \mathrm{CO}_{2}$. The human epithelial cell line HeLa S3 (ATCC CCL-2.2) was grown in DMEM medium supplemented with $10 \%$ FBS, 2 mM L-glutamine, $1 \%$ non-

Table 1. S. cerevisiae Strains Used in this Study

\begin{tabular}{|c|c|c|}
\hline Isolate & Reference & Source \\
\hline S. cerevisiae $60^{(\mathrm{a}, \mathrm{b})}$ & D07.10GE/99 & Clinical isolate (vagina) \\
\hline S. cerevisiae $20^{(\mathrm{a}, \mathrm{b})}$ & G18.19CG/98 & Clinical isolate (faeces) \\
\hline S. cerevisiae $10431^{(\mathrm{b})}$ & CECT 10431 & Spanish natural wine \\
\hline S. cerevisiae var. boulardii $108^{(a, b)}$ & Ultralevura $^{(\mathrm{d})}$ Lot R-08 & Commercial probiotic preparation (UPSAMEDICA S.L., Biocodex) \\
\hline
\end{tabular}

a) Isolates previously collected by the Microbiology Service of the Vall d'Hebron Hospital (Barcelona, Spain) and characterized by De Llanos et al. (2004) [17].

(b) Isolates previously characterized on the basis of potential virulence factors by De Llanos et al. (2006) [23].

(c) Isolate obtained at the Instituto de Agroquímica y Tecnología de Alimentos (CSIC, Spain) (unpublished results).

${ }^{\text {(d) }}$ Commercial name. 
essential amino acids, and $1 \%$ penicillin/streptomycin stock solution (Gibco, Barcelona, Spain) at $37{ }^{\circ} \mathrm{C}$ in $5 \% \mathrm{CO}_{2}$.

For adherence assays, cells were grown up to the formation of a confluent monolayer on a six-well tissue culture plate. After removal of the culture medium, 100 starved yeast cells in $1 \mathrm{ml}$ HBSS (Hanks' balanced salt solution, Gibco, Barcelona, Spain) were added to each well. Yeast cells were previously quantified by microscopic counting and properly diluted in HBSS. Following incubation of cocultures for $45 \mathrm{~min}$ at $37{ }^{\circ} \mathrm{C}$ in $5 \% \mathrm{CO}_{2}$, the non-adhered yeast cells were removed by aspiration of the supernatant, and each well was rinsed twice with $5 \mathrm{ml}$ HBSS. Each well was then overlaid with molten Sabourauddextrose agar at $45^{\circ} \mathrm{C}$, and the plate incubated $24-48 \mathrm{~h}$ at 28 ${ }^{\circ} \mathrm{C}$ for colony development. The number of cfu was determined and adherence is expressed as the percentage (\%) of the adhered cells. Total, adhered and non-adhered, cells $(100 \%)$, were determined as cfu in control plates: $1 \mathrm{ml}$ HBSS containing the yeast suspension used for the adherence assays was plated, overlaid with molten Sabouraud-dextrose agar at $45{ }^{\circ} \mathrm{C}$, and incubated $24-48 \mathrm{~h}$ at $28{ }^{\circ} \mathrm{C}$ for colony development. Quadruplicate determinations were performed for each assay.

Measurement of In Vitro Cytokine Production. The murine macrophage cell line RAW 264.7 (ATCC TIB-71) was used for in vitro assays of TNF- $\alpha$ production in response to both viable and inactivated yeast cells. RAW cells were routinely cultured in RPMI cell culture medium supplemented with 5\% FBS and $1 \%$ penicillin/streptomycin stock solution (Gibco, Barcelona, Spain) at $37^{\circ} \mathrm{C}$ in $5 \% \mathrm{CO}_{2}$. For coculture assays, macrophages were resuspended in the same medium and plated in a 96-well tissue culture plate (1 x $10^{5}$ cells in $200 \mu$ leach well) and incubated for $2 \mathrm{~h}$ at 37 ${ }^{\circ} \mathrm{C}$ in $5 \% \mathrm{CO}_{2}$, prior to the challenge. Starved viable yeast cells $\left(\begin{array}{lllll}1 & \mathrm{x} & 10^{5}\end{array}\right)$ or inactivated yeasts $\left(2 \times 10^{5}\right)$ were resuspended in the same cell culture medium and added (10 $\mu 1$ each well) to macrophages to give a $1 / 1$ or $1 / 2$ ratio (macrophage/yeast) respectively. Cocultures were incubated for $6 \mathrm{~h}$ at $37{ }^{\circ} \mathrm{C}$ in $5 \% \mathrm{CO}_{2}$. Assays in the absence of exogenous stimuli were used as negative controls to check background activation. Supernatants were then harvested and tested for TNF- $\alpha$ using a commercial enzyme-linked immunosorbent assay (ELISA) (eBioscience, San Diego, USA) as previously described $[35,41,42]$. Triplicate determinations were performed in each assay.

Proliferation of yeast cells and viability of macrophages were also determined after cocultures. Viable yeasts were determined as cfu by plating serial dilutions from each well (before and after coculture) on YPD plates; result is expressed as the percentage (\%) of viable yeasts after $6 \mathrm{~h}$ of coculture. Viability of macrophages was determined by flow cytometry; briefly, after $2 \mathrm{~h}$ of coculture, macrophages ( $1 \mathrm{x}$ $10^{5}$ ) were removed from wells, resuspended in $100 \mu$ PBS and labelled with $0.5 \mu \mathrm{g} / \mathrm{ml}$ fluorescein isothiocynate (FITC)-anti F4/80 mAb (clone BMB8, eBioscience, San Diego, USA) for $20 \mathrm{~min}$ at $4{ }^{\circ} \mathrm{C}$, and then washed with PBS and added with propidium iode $(10 \mu \mathrm{g} / \mathrm{ml})$ (Sigma-Aldrich, Madrid, Spain); non-viable macrophages (labelled with both FITC-anti F4/80 and propidium iode) were determined by flow cytometry analysis with an EPICS XL-MCL flow cytometer (Beckman Coulter).
Phagocytosis Assays. Viable yeast cells were labelled with FITC according to standard procedures [35]. Briefly, yeast were resuspended in carbonate buffer $\mathrm{pH} 10\left(5 \times 10^{6}\right.$ cells $/ \mathrm{ml}$ ) containing $100 \mu \mathrm{g}$ of FITC per $\mathrm{ml}$, and incubated at room temperature for $2 \mathrm{~h}$. Soluble FITC was removed by extensive washing with PBS, and homogeneous distribution of FITC labelling was checked by flow cytometry. Macrophages (RAW 264.7 cells) resuspended in supplemented RPMI medium with antibiotics (see above) were plated in a 96-well tissue culture plate $\left(1 \times 10^{5}\right.$ cells in $200 \mu \mathrm{l}$ each well), added with FITC-labelled yeast cells (5 x $10^{5}$ cells in $20 \mu$ l of RPMI medium each well) and incubated for $30 \mathrm{~min}$ at $37{ }^{\circ} \mathrm{C}$ in $5 \% \mathrm{CO}_{2}$. Macrophages were labelled with $0.25 \mu \mathrm{g} / \mathrm{ml}$ phycoerythrin (PE)-anti F4/80 mAb (clone $\mathrm{BM} 8$, eBioscience, San Diego, USA) for $20 \mathrm{~min}$ at $4{ }^{\circ} \mathrm{C}$ and then washed with PBS, as previously described [44]. The extent of phagocytosis was assessed as the percentage of cells both red- and green-labelled by flow cytometry analysis with an EPICS XL-MCL flow cytometer (Beckman Coulter). To differentiate cell surface-adherent yeasts from the internalised ones, trypan blue $(0.2 \%)$ was added to quench extracellular fluorescence. Triplicate determinations were performed in each assay.

Statistical Analysis. Survival curves were analysed by Kaplan-Meyer log rank test. The two tailed $t$-test was used to analyse results of fungal burden in organs, cytokine production, adhesion and phagocytosis between individual yeast strains by pair comparisons. Significance was accepted at $P<0.05(*)$ and $P<0.01(* *)$.

\section{RESULTS}

\section{Virulence of $S$. cerevisiae Strains in a Mouse Model of Infection}

As susceptibility to fungal infections may vary among mouse strains, we performed the study of the virulence of $S$. cerevisiae strains using three different inbred strains: DBA $/ 2, \mathrm{C} 3 \mathrm{H} / \mathrm{HeN}$ and $\mathrm{C} 57 \mathrm{BL} / 6$. Survival curves of animals $(\mathrm{n}=8)$ i.v. injected with $2 \times 10^{7}$ yeasts were similar in all cases (not shown). C57BL/6 mice were resistant to all yeast strains tested, as no animal died during the assay. The other two mouse strains (DBA/2 and $\mathrm{C} 3 \mathrm{H} / \mathrm{HeN})$ showed an identical behaviour, as only one mouse died when animals were injected with the S. cerevisiae D14 strain. Therefore, no statistical differences were found in survival concerning both mouse and yeast strains. Interestingly, all three mouse strains showed temporary but clear symptoms of infection (lethargy, ruffled appearance), particularly in response to S. cerevisiae strains 60, 61 and D14, suggesting differences of virulence among yeast strains. Such differences were demonstrated when fungal burden in organs was determined in DBA/2 mice. As shown in Fig. (1), at day 7 post-infection the faecal isolate 20 and the laboratory wine yeast CECT 10431 showed very low levels of fungal burden in the organs analysed. S. cerevisiae strains 60, 61 and D14 showed significant levels of fungal burden in brain, whereas $S$. boulardii showed an intermediate colonization (Fig. 1A). Strain D14 of S. cerevisiae showed detectable capability to invade the kidney, whereas $S$. cerevisiae 60,61 and $S$. boulardii showed a more limited ability to colonize kidneys, with no statistical difference as compared to the $S$. cerevisiae CECT 10431 strain and the faecal isolate 20 (Fig. 1B). S. 


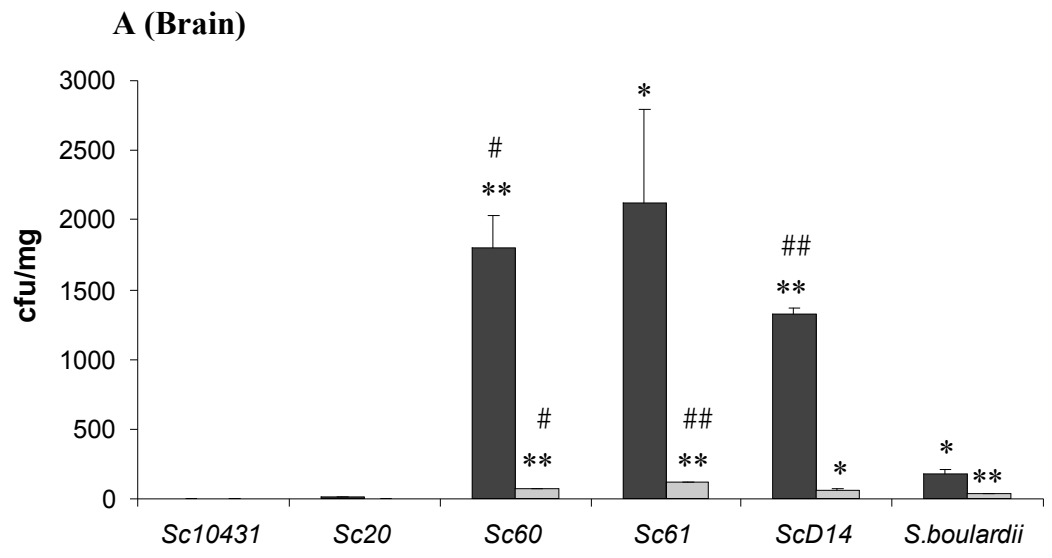

\section{B (Kidney)}

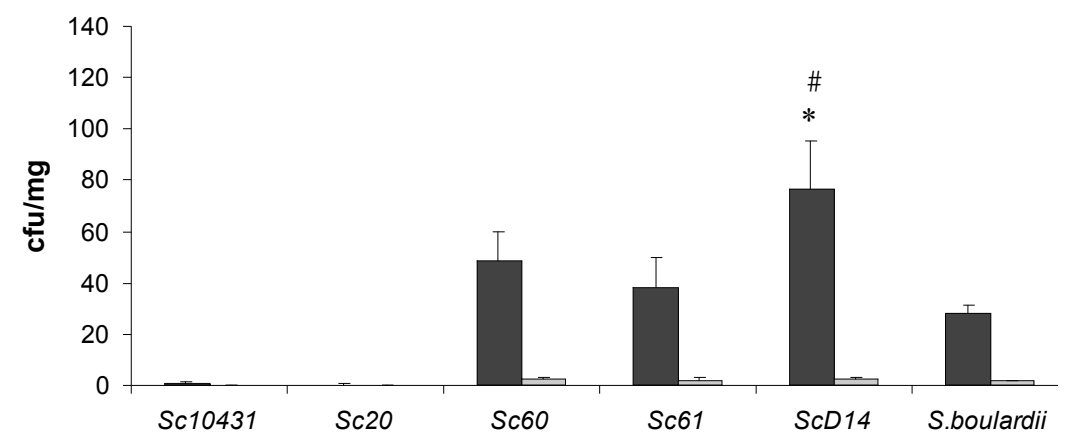

\section{C (Spleen)}

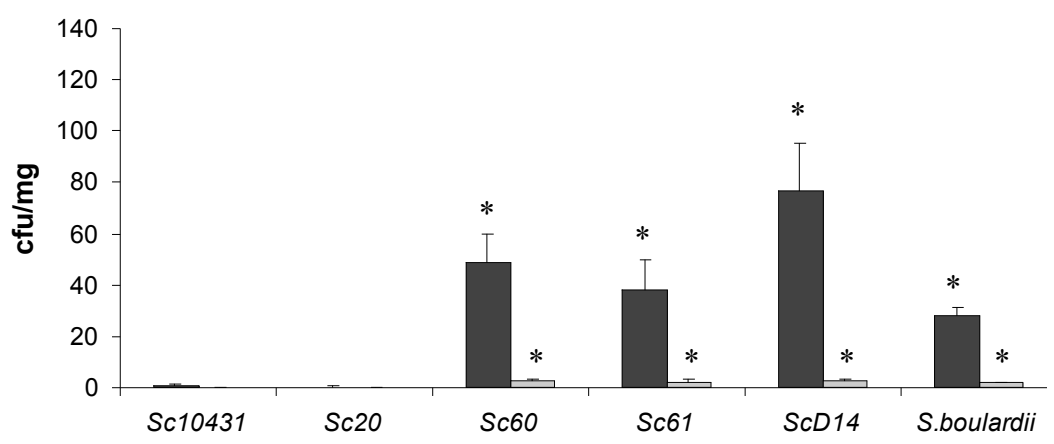

Fig. (1). Fungal burden in organs from mice experimentally infected with yeast isolates. DBA/2 mice $(\mathrm{n}=14)$ were i.v. injected with $2 \times 10^{7}$ yeast cells of S. cerevisiae CECT 10431 (Sc10431), S. cerevisiae clinical isolates 20 (faecal), 60 and 61 (vaginal) (Sc20, Sc60 and Sc61, respectively), $S$. cerevisiae D14 strain (ScD14), and S. boulardii. At day 7 (black bars) and 14 (grey bars) post-infection the tissue outgrowth of yeasts was determined in homogenized organs from three animals: brain (A), kidney (B) and spleen $(\mathbf{C})$. Results are expressed as mean values of cfu $/ \mathrm{mg} \pm$ standard deviation from one representative assay of two. ${ }^{*} P<0.05,{ }^{* *} P<0.01$ when strains are individually compared to either $S$. cerevisiae CECT 10431 strain or the faecal isolate 20 (statistical significance are identical for both comparisons). ${ }^{\#} P<0.05$, ${ }^{\# \#} P$ $<0.01$ when 60,61 and D14 strains are individually compared to $S$. boulardii. All other comparisons between yeast strains are statistically no significant.

cerevisiae 60, 61, D14 and S. boulardii strains showed a limited, but significant ability to colonize the spleen when compared to the faecal isolate 20 and the CECT 10431 $S$. cerevisiae strains (Fig. 1C). In agreement with the lack of mortality, at day 14 post-infection fungal burden diminished in all cases, indicating that the clearance of the microorganisms occurs in immunocompetent mice. Similar results were obtained when fungal burden in organs was studied in $\mathrm{C} 3 \mathrm{H} / \mathrm{HeN}$ mice (not shown). These results point out that some Saccharomyces strains, from both clinical and non-clinical (dietetic and probiotic/biotherapeutic) origin, possess the ability to infect organs, with special affinity for the brain. 


\section{Adhesion of $S$. cerevisiae Strains to Epithelial and Endothelial Cells}

As adhesion to host tissues is a key step to establish the infectious process and therefore it is considered as a fungal virulence attribute [19,32], we investigated the ability of yeast strains to adhere in vitro to epithelial and endothelial cell monolayers. In these assays two C. albicans strains were used as controls: the high virulence ATCC 26555 strain, and the low virulence PCA2 strain.

Assays of adherence to endothelial ECV304 cells showed that Candida strains possess a much higher adhesion ability than Saccharomyces isolates: about $65 \%$ and $30 \%$ for $C$. albicans ATCC 26555 and PCA2 strains, respectively (Fig. 2A). Adherence of Saccharomyces isolates was hetero-

\section{A (ECV304)}

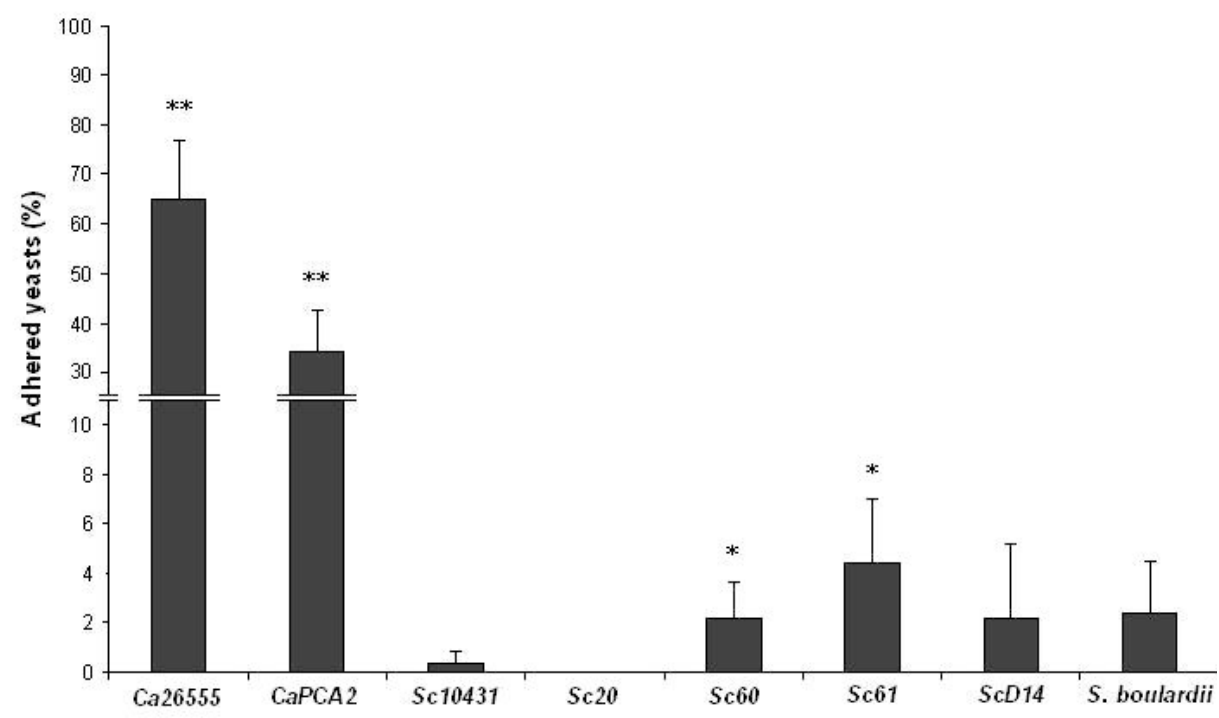

\section{B (HeLa S3)}

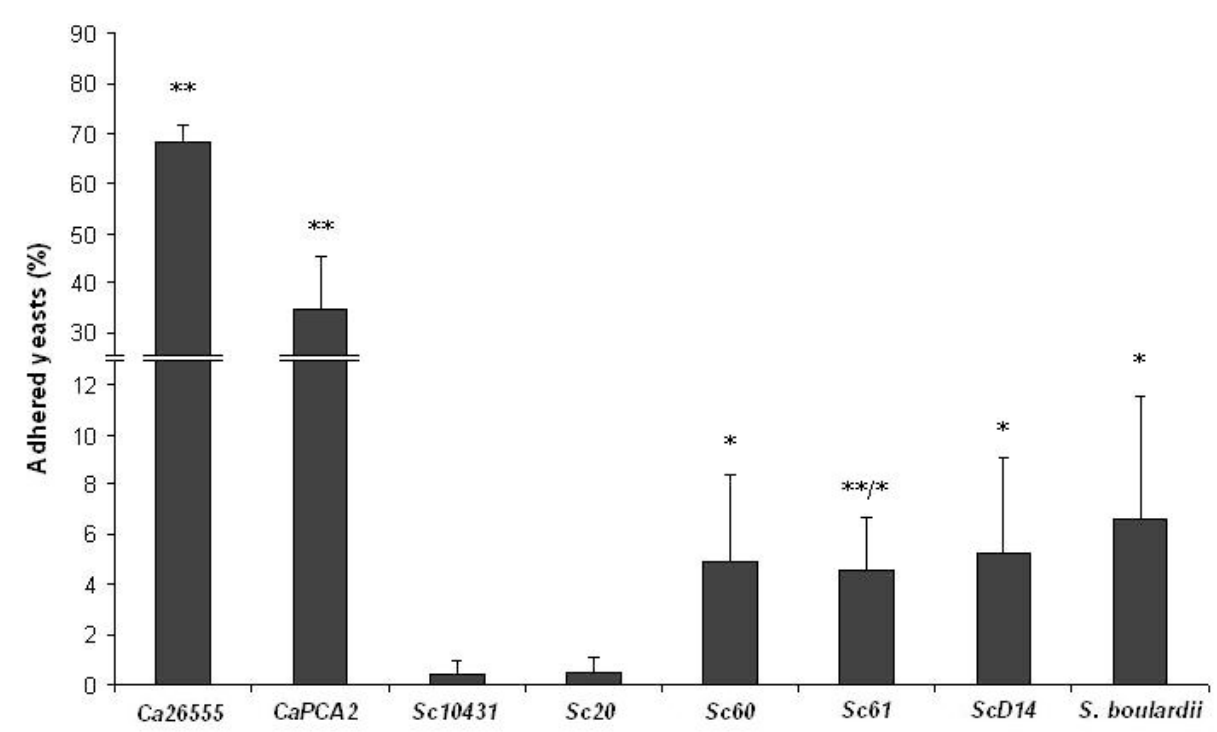

Fig. (2). Adhesion of yeasts to cell culture monolayers. Yeast cells (100 cfu) of tested strains (S. cerevisiae CECT 10431, S. cerevisiae clinical isolates 20, 60 and 61, S. cerevisiae D14 strain, S. boulardii, named as in Fig. (1) legend, and C. albicans ATCC 26555 and PCA2 strains) were added to plates containing monolayer cultures of the endothelial ECV304 cell line (A) and the epithelial HeLa S3 cell line (B). After 45 min of incubation at $37{ }^{\circ} \mathrm{C}$ in $5 \% \mathrm{CO}_{2}$ and extensive washing, molten Sabouraud-dextrose agar was added and incubated at $37{ }^{\circ} \mathrm{C}$ for $24-48 \mathrm{~h}$ for colony development of adhered yeasts. Results are expressed as the percentage of adhered yeast cells and data represent mean values \pm standard deviation of quadruplicates from one representative assay of two. ${ }^{*} P<0.05,{ }^{* *} P<0.01$ when strains are individually compared to either $S$. cerevisiae CECT 10431 strain or the faecal isolate 20 (differences in statistical significance for both comparisons are indicated: Sc10431/Sc20). 
geneous among isolates: vaginal isolate 61 showed the highest adherence (4\%), vaginal isolate 60 , dietetic strain D14 and $S$. boulardii showed a minor adherence ability (2 $\%$ ), whereas the laboratory CECT 10431 and the faecal 20 strains showed the lowest adherence levels $(0.5 \%$ and nondetectable, respectively). Differences between vaginal isolates (60 and 61) and strains CECT 10431 and 20 were statistically significant. As expected, differences between all $S$. cerevisiae strains and $C$. albicans strains were also statistically significant (not shown). These results indicate that Saccharomyces isolates, both clinical and non-clinical, may possess a limited but significant adherence ability to endothelial cells.

Results of yeast adherence to epithelial HeLa S3 cells were more homogeneous (Fig. 2B). Again Candida strains showed a good ability of adhesion (70 \% and $35 \%$ for C. albicans ATCC 26555 and PCA2 strains, respectively). Vaginal isolates 60 and 61 and non-clinical D14 and $S$. boulardii strains showed similar adherence ability (5-6\%), whereas strains 20 and CECT 10431 showed only a residual adherence $(<0.5 \%)$, and these differences were statistically significant. As expected, differences between all $S$. cerevisiae isolates and $C$. albicans strains were also statistically significant (not shown). These results confirm that clinical and non-clinical Saccharomyces isolates possess detectable adherence properties that can be envisaged as a potential virulence factor.

\section{In vitro Cytokine Production by Macrophages in Response to $S$. cerevisiae Strains}

Production of proinflammatory cytokines, such as TNF$\alpha$, upon recognition of microbial ligands by immune cells plays a key role in host protection against infection, and modulation of this response by the pathogen may favour the progress of the infectious process, as described for the fungal species $C$. albicans [20, 31, 45]. Therefore, we studied the ability of yeast cells to induce in vitro the production of TNF- $\alpha$ by the macrophage cell line RAW 264.7. Macrophages were challenged with viable yeast cells for $6 \mathrm{~h}$ to allow production of detectable levels of cytokine. Results (Fig. 3A) showed that total production of cytokine was similar in response to all yeast stimuli used, including $C$. albicans PCA2 strain, except for $S$. cerevisiae CECT 10431 which induces lower levels of TNF- $\alpha$ production. Negative controls without exogenous stimuli resulted in the production of non-detectable cytokine levels.

As viable yeast cells were used as stimuli, several factors may influence cytokine production, such as proliferation of yeast population during the assay and viability of macrophages after challenging. Therefore, we determined the ability of yeast isolates to proliferate during coculture with macrophages. As shown in Fig. (3B), the total C. albicans PCA2 population increased 5-fold, whereas the number of yeast cells strongly decreased in the case of the $S$. cerevisiae CECT 10431 and remained almost unchanged for $S$. cerevisiae 20; the yeast population of the other four $S$. cerevisiae isolates increased about 2- to 4-fold. Differences between strains were statistically significant when individually compared to both $S$. cerevisiae CECT 10431 and 20 strains (Fig. 3B). This indicates that yeast strains possess a different ability to proliferate under the conditions of the assay, therefore modifying the amount of fungal challenge during coculture assays. Fig. (3C) shows the relative production of TNF- $\alpha$ standardized according to the final yeast population, although these data may overestimate the effect of fungal growth. C. albicans PCA2 strain showed the lower levels of relative cytokine production, whereas strains S. cerevisiae CECT 10431 and 20 induced the highest levels of cytokine production, and the other four Saccharomyces isolates showed intermediate levels. Differences between strains were statistically significant when individually compared to both $S$. cerevisiae CECT 10431 and 20 strains (Fig. 3C). These differences among Saccharomyces isolates were also statistically significant when macrophages were challenged with inactivated yeast stimuli (Fig. 4); interestingly differences between $S$. cerevisiae 60, 61, D14 and $S$. boulardii strains individually compared to $C$. albicans PCA2 were statistically significant (not shown).

Viability of RAW cells was also determined after $2 \mathrm{~h}$ of coculture with viable yeast stimuli (Fig. 5). All Saccharomyces strains caused similar mortality rates $(2-5 \%$ of non-viable RAW cells), except strain 60 which caused an increased mortality rate $(11 \%)$. Differences between strain 60 and both CECT 10431 and 20 strains were statistically significant. The low virulence $C$. albicans PCA2 strain induced intermediate levels of non-viable macrophages (6-7 $\%$ ), whereas the high virulence C. albicans ATCC 26555 strain caused an increased mortality rate (19\%), statistically significant when compared to all other strains, including the 60 isolate.

\section{Phagocytosis of $\boldsymbol{S}$. cerevisiae Strains by Macrophages}

The above described results point out that phagocytosis of viable yeast cells may also vary among strains. The ability of RAW cells to phagocytose yeast cells was determined by flow cytometry analysis after $30 \mathrm{~min}$ of coculture with FITC-labelled yeast. Results (Fig. 6) indicated that the laboratory reference $S$. cerevisiae CECT 10431 strain is the best phagocytosed yeast (about $60 \%$ of macrophages were able to engulf yeast cells). Commensal S. cerevisiae 20,60 and 61 isolates showed similar phagocytose rates (33-46\%) as well as C. albicans PCA2 (39\%) and ATCC 26555 (29\%) strains. Interestingly, dietetic and biotherapeutic yeast strains (D14 and $S$. boulardii) were poorly phagocytosed (19\% and $9 \%$ respectively), and these differences were statistically significant when $S$. boulardii was compared to all commensal and C. albicans strains (Fig. 6), and when ScD14 was compared to C. albicans PCA2 and S. cerevisiae 60 and 61 strains (not shown).

\section{DISCUSSION}

Molecular characterization of $S$. cerevisiae clinical isolates has demonstrated their association with non-clinical strains, and therefore exposure due to the widespread handling by consumers of probiotic and baker's yeast may originate human colonization $[10,17,18]$. Potential virulence factors, such as secretion of higher levels of hydrolytic enzymes (proteases and phospholipases), the ability to grow at $42{ }^{\circ} \mathrm{C}$ and to develop pseudohyphal growth are frequently associated with clinical isolates and have been proposed as preventive criteria in the selection for industrial strains [23]. However, no specific virulence factor has been found that 
A

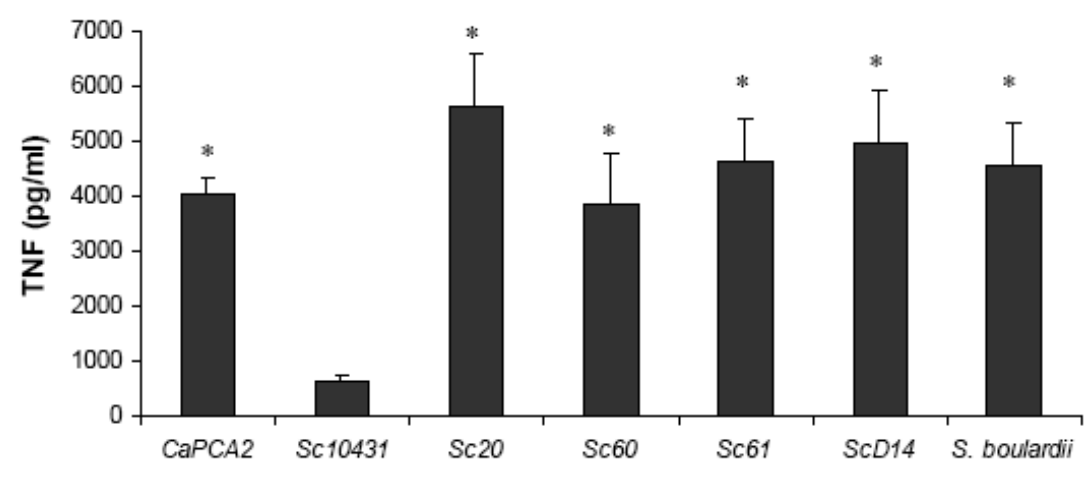

B

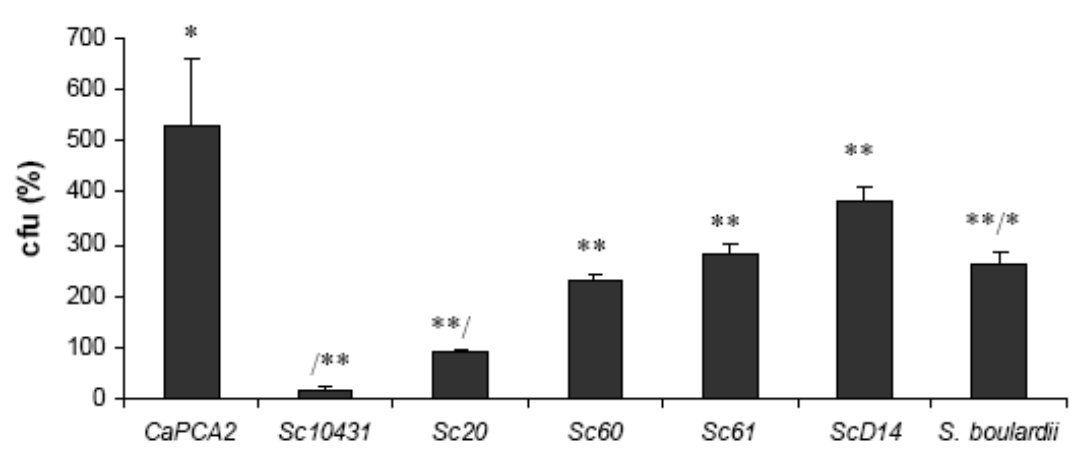

C

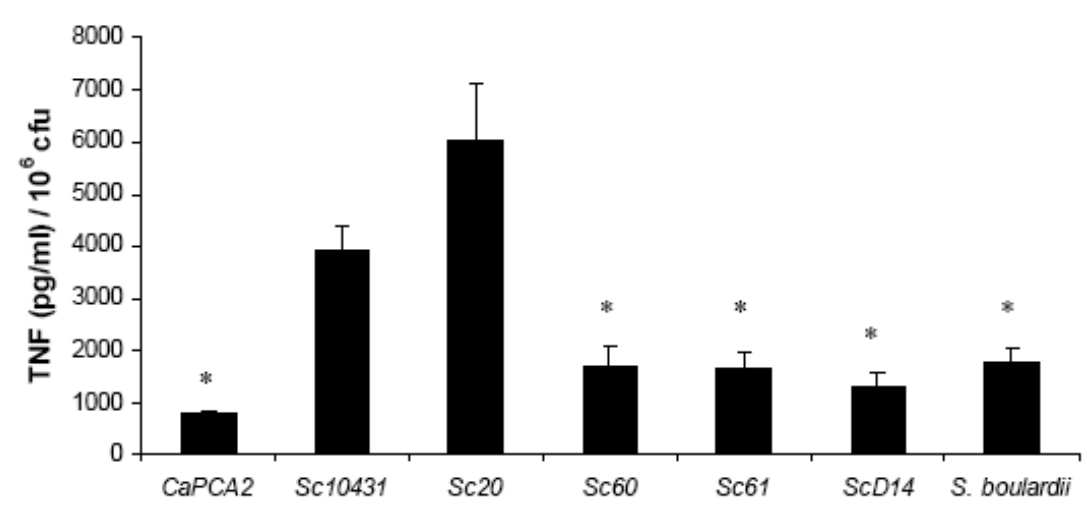

Fig. (3). Production of TNF- $\alpha$ by macrophages in response to viable yeasts. RAW 264.7 macrophages $\left(1 \times 10^{5}\right.$ cells) were cocultured with viable yeast $\left(1 \times 10^{5}\right.$ cells) of the tested strains (S. cerevisiae CECT 10431, S. cerevisiae clinical isolates 20, 60, and 61, S. cerevisiae D14 strain, $S$. boulardii, and C. albicans PCA2 strain), and incubated for $6 \mathrm{~h}$ at $37{ }^{\circ} \mathrm{C}$ in $5 \% \mathrm{CO}_{2}$. (A) The concentration of TNF- $\alpha$ in the culture supernatants was measured by ELISA. Results are shown as means \pm standard deviation of triplicates from one representative assay of two. (B) Proliferation of yeasts after coculture. Viable yeasts were determined as cfu by plating serial dilutions on YPD plates (before and after coculture), and results are expressed as the percentage (\%) of viable cells \pm standard deviation of triplicates from one representative assay of two. (C) Relative production of TNF- $\alpha$ by macrophages in response to viable yeast. Total production of TNF- $\alpha$, shown in panel A, was standardized according to the final yeast population. Results are expressed as the ratio TNF- $\alpha$ production $/ 10^{6}$ cfu \pm standard deviation of triplicates from one representative assay of two. ${ }^{*} P<0.05, * * P<0.01$ when strains are individually compared to $S$. cerevisiae CECT 10431 (A), and compared to either S. cerevisiae CECT 10431 strain or the faecal isolate 20 (Sc10431/Sc20) (B, C).

clearly separates the strains from clinical and non-clinical origin [30]. Since in vivo and in vitro experimental approaches to study interaction of fungal cell with the host have been a very useful tool to elucidate molecular mechanism leading to infection by $C$. albicans $[20,31,35$, $36,45]$, in this work we have studied the interactions of six $S$. cerevisiae (three clinical and three non-clinical) isolates from different origins (two commensal isolates from vagina, one commensal isolate from faeces, two strains from dietetic and probiotic products, and one reference wine strain) with the host, in order to gain information about virulence-related factors contributing to the infectious process and their association with clinical and/or non-clinical isolates. 


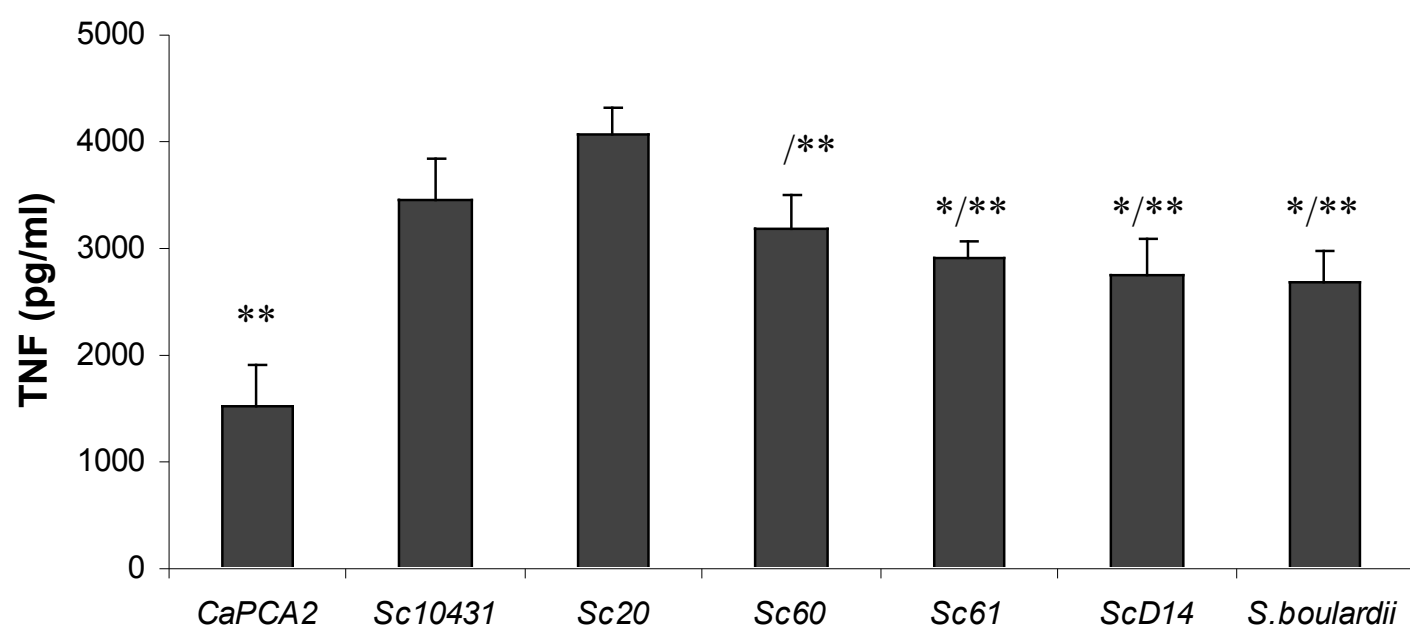

Fig. (4). Production of TNF- $\alpha$ by macrophages in response to inactivated yeasts. RAW 264.7 macrophages $\left(1 \mathrm{x} 10^{5}\right.$ cells $)$ were cocultured with inactivated yeasts $\left(2 \times 10^{5}\right.$ cells) of the tested strains (S. cerevisiae CECT 10431, S. cerevisiae clinical isolates 20, 60, and 61, S. cerevisiae D14 strain, $S$. boulardii and C. albicans PCA2 strain), and incubated for $6 \mathrm{~h}$ at $37{ }^{\circ} \mathrm{C}$ in $5 \% \mathrm{CO}_{2}$. The concentration of TNF- $\alpha$ in the culture supernatants was measured by ELISA. Results are shown as means \pm standard deviation of triplicates from two representative assays. ${ }^{*} P<0.05, * * P<0.01$ when strains are individually compared to either $S$. cerevisiae CECT 10431 strain or the faecal isolate 20 (Sc10431/Sc20).

In vivo models of systemic infection in mouse strains demonstrate that all $S$. cerevisiae isolates possess a very limited virulence potential, as a high i.v. dose of yeast $\left(20 \times 10^{7}\right)$ did not result in significant mortality of immunocompetent animals in all three mouse strains tested. It should be noted that i.v. administration of one million of C. albicans yeast usually results in a significant mortality of animals in a few days $[35,36,39]$. Therefore, pathogenicity of $S$. cerevisiae isolates is far too low to be comparable to $C$. albicans pathogenic potential. Nevertheless, analysis of fungal burden during infection revealed some interesting aspects. Clinical (vaginal) and non-clinical (dietetic and biotherapeutic) isolates showed a clear ability to invade organs, preferentially the brain, of immunocompetent mice, which may account for the symptoms observed in infected animals. By contrast, both the faecal isolate and the reference wine strain, which differ in the expression of potential virulence factors, were unable to invade these organs, pointing out that virulence and expression of potential virulence traits do not correlate necessarily. The clearance of fungal burden during infection explains the absence of mortality of animals and further suggests a limited pathogenicity of isolates in immunocompetent mice. It is remarkable that one dietetic isolate (D14) showed a high ability to invade organs (including kidney) and was responsible for the only two deaths observed in animals, suggesting a higher virulence potential.

We also determined the adherence properties of the isolates to cell cultures, as adhesion to host tissues allows colonization, a key step to establish the infectious process $[19,32]$. Results showed that both clinical (vaginal) and nonclinical (dietetic and biotherapeutic) isolates possess a limited, but detectable ability to adhere to endothelial, and particularly to epithelial cell lines, as compared to the faecal

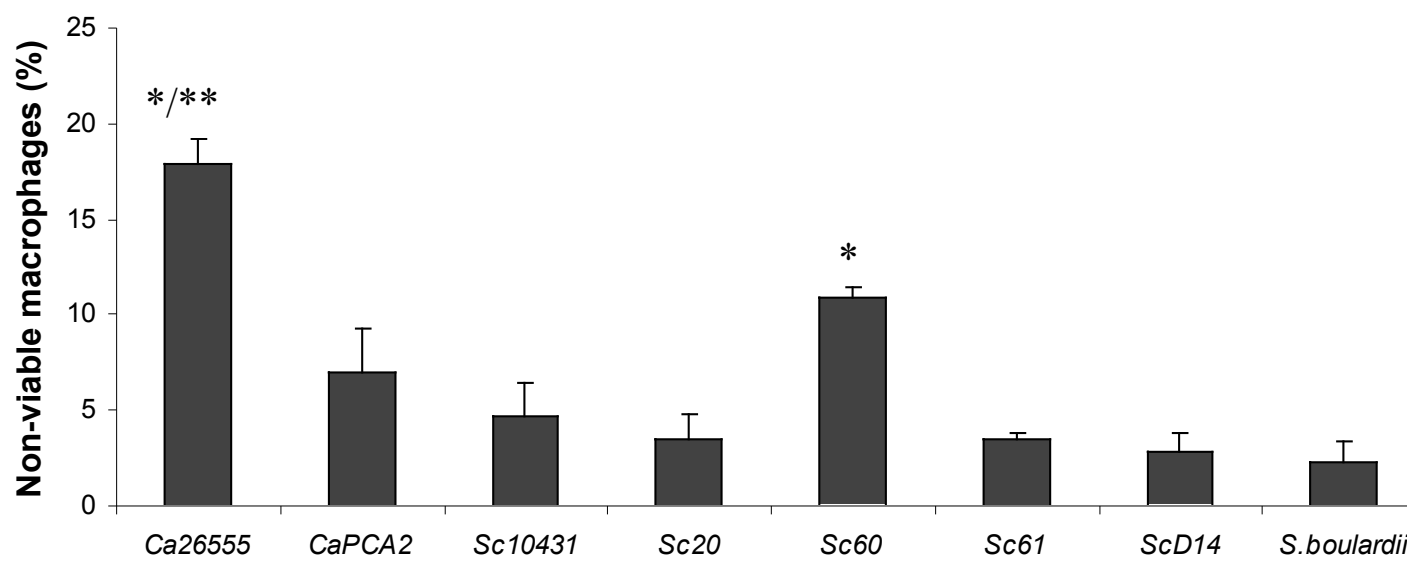

Fig. (5). Viability of macrophages after coculture with viable yeasts. RAW 264.7 macrophages were cocultured with viable yeast cells (1:1 ratio) for $2 \mathrm{~h}$ at $37{ }^{\circ} \mathrm{C}$ in $5 \% \mathrm{CO}_{2}$. Macrophages were labelled with FITC anti-F4/80 mAb, and non-viable macrophages were stained with propidum iode and quantified by flow cytometry analysis. Strains assayed are named as in Fig. (2). Data represent the percentage of nonviable cells \pm standard deviation of triplicates from one representative assay of two. ${ }^{*} P<0.05,{ }^{*} P<0.01$ when strains are individually compared to either $S$. cerevisiae CECT 10431 strain or the faecal isolate 20 (Sc10431/Sc20). 


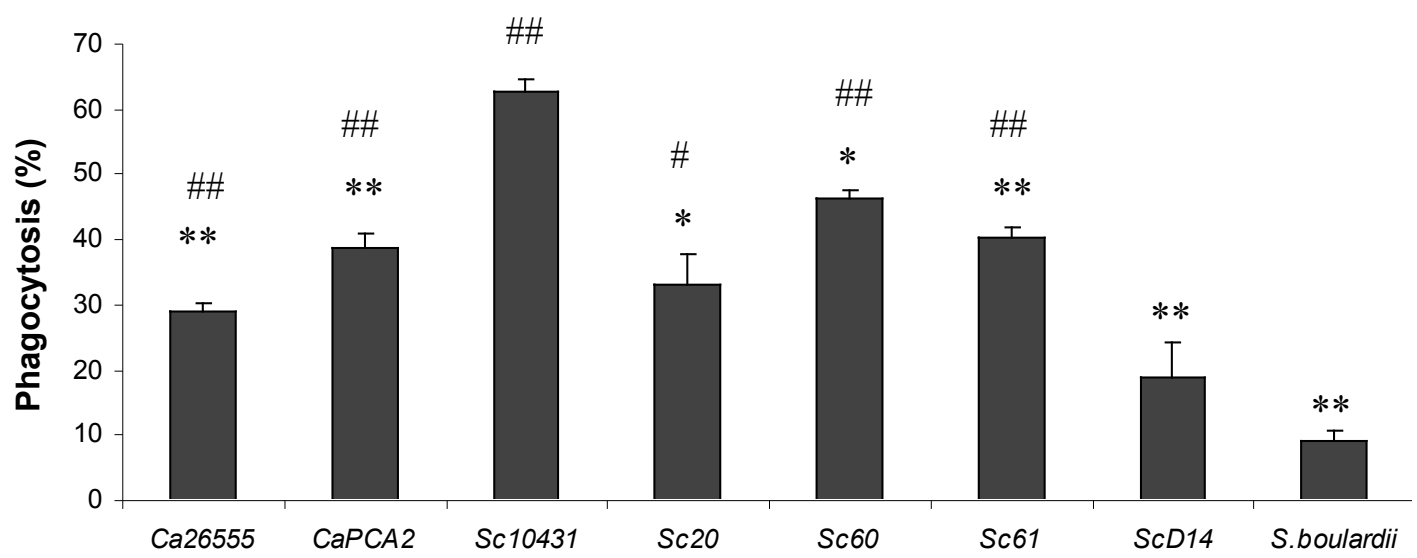

Fig. (6). Phagocytosis of yeast isolates by macrophages. RAW 264.7 macrophages were cocultured with FITC-labelled viable yeasts (ratio 1:5) for $30 \mathrm{~min}$ at $37{ }^{\circ} \mathrm{C}$ in $5 \% \mathrm{CO}_{2}$. Macrophages were labelled with PE-anti $\mathrm{F} 4 / 80 \mathrm{mAb}$ and the extent of phagocytosis was assessed by flow cytometry analysis. Strains assayed are named as in Fig. (2). Data represent the percentage of macrophages containing internalised yeast \pm standard deviation of triplicates from one representative assay of two. ${ }^{*} P<0.05,{ }^{*} P<0.01$ when strains are individually compared to $S$. cerevisiae CECT 10431 strain. ${ }^{\#} P<0.05$, ${ }^{\# \# ~} P<0.01$ when individual strains are individually compared to $S$. boulardii.

isolate and the reference wine strain, which were much less adherent, and to $C$. albicans, which possess a higher adherence potential. A similar result was reported for the adhesion of $S$. cerevisiae clinical and non-clinical isolates to Caco-2 and buccal epithelial cells [30, 46]. This ability to adhere to host cells can be considered as a virulence factor contributing to the pathogenicity of $S$. cerevisiae isolates.

Proinflammatory response to invading pathogens plays an essential role in host protection, and modulation of this response by the pathogen may contribute to development of disease, as demonstrated to occur in response to C. albicans; production of proinflammatory cytokines, such as TNF- $\alpha$, is triggered upon recognition of well conserved fungal ligands by PRRs on immune cells, and expression and accessibility of these ligands may vary among strains [20, 31, 35, 36, 45]. In vitro production of TNF- $\alpha$ by macrophages in response to inactivated $S$. cerevisiae isolates showed significant differences among strains, with the highest production of cytokine observed in response to the faecal isolate and the reference $S$. cerevisiae wine strain. Results of TNF- $\alpha$ production in response to viable yeasts confirm this observation, although they are more difficult to interpret since several factors should be considered, as already mentioned in the Results section. Our data indicate that the relative production of TNF- $\alpha$ is lower in clinical (vaginal) and non-clinical (dietetic and biotherapeutic) $S$. cerevisiae strains as compared to the faecal isolate and the reference strain, although higher than cytokine production in response to $C$. albicans PCA2. As TNF- $\alpha$ is an essential cytokine for host protection against fungal infections [20, 31], it can be suggested that the lower relative levels of cytokine production observed in those strains may probably contribute to their increased virulence. In addition, viable yeasts may possess properties able to modulate interaction with macrophages. This is supported by the observed ability of clinical (vaginal) and non-clinical (dietetic and biotherapeutic) isolates of $S$. cerevisiae to proliferate in the presence of macrophages, although viability of RAW cells was only significantly decreased in response to the clinical isolate 60. Moreover, non-clinical (dietetic and biotherapeutic) strains showed a decreased phagocytosis rate as compared to clinical and reference isolates; this observation is of interest as destruction of microbes by phagocytes is the main defence against primary infection by pathogenic yeast species, such as $C$. albicans $[20,31]$. Therefore, an increased resistance to phagocytosis may also contribute to the pathogenesis of dietetic and probiotic strains. Our results point out that yeast strains, such as $S$. boulardii and D14, present in probiotic and dietetic commercial products, are able to express in vitro some traits that can be considered as putative virulence factors, and therefore the widespread handling of these products by the consumers must be cautiously regarded, as it may cause human colonization and/or transmission of infection by touch contamination in immunocompromised patients.

As above mentioned, the ability of $S$. cerevisiae strains to invade the host does not necessarily correlate with the expression of potential virulence traits in vitro. The differences observed among $S$. cerevisiae strains concerning interaction with host (adhesion, cytokine production and phagocytosis) may be partly due to differential expression of surface molecules which specifically interact with host receptors. Composition and organization of $S$. cerevisiae cell wall is similar to that of $C$. albicans, made basically of chitin, glucan, and mannoproteins, and therefore it is assumable that both species may share some ligands involved in host interaction [32, 47-49]. In fact, the yeast cell wall particle Zymosan is recognized by host PRRs, such as dectin-1 and TLR2, which also recognize $C$. albicans, and play a key role in host defences against candidiasis [20, 31$37,45]$. It should be noted that the expression of these ligands, as well as the expression of virulence factors in $S$. cerevisiae, may be strain dependent and also environmentally regulated, as does occur in $C$. albicans $[19,32]$, and therefore one single isolate may express putative virulence factors depending on growth conditions. Further studies are required to identify particular virulence factors in $S$. cerevisiae isolates and to determine their expression and role in virulence.

In conclusion, our results supports the notion that clinical and non-clinical (dietetic and probiotic) $S$. cerevisiae isolates 
may share some properties in their relationship with the host (such as adhesion to host tissues, resistance to phagocytosis and the ability to modulate the proinflammatory response) that contribute to the infectious process. These properties can be considered as new putative virulence factors that should be added to the already described (growth at $42{ }^{\circ} \mathrm{C}$, pseudohyphal development, and production of proteases and phospholipases). Therefore, we propose that selection criteria for industrial strains should also include studies concerning host-pathogen interactions, such as adhesion to cell lines, in vitro cytokine production and phagocytosis, in order to select safe strains.

\section{ACKNOWLEDGEMENTS}

A. Yáñez, C. Murciano and S. Llopis are recipients of fellowships from the Ministerio de Educación y Ciencia, Spain. We thank Universitat de València and SCSIE (Universitat de València) for financial and technical support.

\section{REFERENCES}

[1] McFarland LV, Surawicz CM, Greenberg RN, et al. A randomised placebo-controlled trial of Saccharomyces boulardii in combination with standard antibiotics for Clostridium difficile disease. J Med Am Assoc 1994; 271: 1913-9.

[2] Doron SI, Hibberd PL, Gorbach SL. Probiotics for prevention of antibiotic-associated diarrhoea. J Clin Gastroenterol 2008; 42 (Suppl 2): 58-63.

[3] Goldin BR, Gorbach SL. Clinical indications for probiotics: an overwiew. Clin Infect Dis 2008; 46 (Suppl 2): 96-100.

[4] Hazen KC. New and emerging yeast pathogens. Clin Microbiol Rev 1995; 8: 462-78.

[5] Murphy A, Kavanagh K. Emergence of Saccharomyces cerevisiae as a human pathogen: implications for biotechnology. Enzyme Microb Technol 1999; 25: 551-7.

[6] Pontón J, Rüchel R, Clemons KV, et al. Emerging pathogens. Med Mycol 2000; 38 (Suppl 1): 225-36.

[7] Enache-Angoulvant A, Hennequin C. Invasive Saccharomyces infection: a comprehensive review. Clin Infect Dis 2005; 41: 155968.

[8] Herbrecht R, Nivoix Y. Saccharomyces cerevisiae fungemia: an adverse effect of Saccharomyces boulardii probiotic administration. Clin Infect Dis 2005; 40: 1635-7.

[9] Muñoz P, Bouza E, Cuenca-Estrella M, et al. Saccharomyces cerevisiae fungemia: an emerging infectious disease. Clin Infect Dis 2005; 40: 1625-34.

[10] De Llanos R, Querol A, Pemán J, et al. Food and probiotic strains of Saccharomyces cerevisiae species as a possible origin of human systemic infections. Int J Food Microbiol 2006; 110: 286-9.

[11] Hennequin C, Kaufmann-Lacroix C, Jobert A, et al. Possible role of catheters in Saccharomyces boulardii fungemia. Eur J Clin Microbiol Infect Dis 2000; 19: 16-20.

[12] Lherm T, Monet C, Nougière B, et al. Seven cases of fungemia with Saccharomyces boulardii in critically ill patients. Intensive Care Med 2002; 28: 797-801.

[13] Cassone M, Serra P, Mondello F, et al. Outbreak of Saccharomyces cerevisiae subtype boulardii fungemia in patients neighboring those treated with a probiotic preparation of the organism. J Clin Microbiol 2003; 41: 5340-3.

[14] Graf C, Gavazzi G. Saccharomyces cerevisiae fungemia in an immunocompromised patient not treated with Saccharomyces boulardii preparation. J Infect 2007; 54: 310-1.

[15] Jensen DP, David MD, Smith MD. Fever of unknown origin secondary to brewer's yeast ingestion. Arch Intern Med 1976; 136: 332-3.

[16] Smith D, Metzgar D, Wills C, Fierer J. Fatal Saccharomyces aortic graft infection. J Clin Microbiol 2002; 40: 2691-2.

[17] De Llanos R, Querol A, Planes MP, Fernández-Espinar MT. Molecular characterization of clinical Saccharomyces cerevisiae isolates and their association with non-clinical strains. Syst Appl Microbiol 2004; 27: 427- 35.

[18] MacKenzie DA, Defernez M, Dunn WB, et al. Relatedness of medically important strains of Saccharomyces cerevisiae as revealed by phylogenetics and metabolomics. Yeast 2008; 25: 50112.

[19] Calderone RA. Candida and candidiasis. $2^{\text {nd }}$ ed. New York: American Society for Microbiology 2002.

[20] Zelante T, Montagnoli C, Bozza S, et al. Receptors and pathways in innate antifungal immunity: the implications for tolerance and immunity to fungi. Adv Exp Med Biol 2007; 590: 209-21.

[21] McCusker JH, Clemons KV, Stevens DA, Davis RW. Saccharomyces cerevisiae virulence phenotype as determined with CD-1 mice is associated with the ability to grow at $42{ }^{\circ} \mathrm{C}$ and form pseudohyphae. Infect Immun 1994; 62: 5447-55.

[22] McCullough MJ, Clemons KV, McCusker JH, Stevens DA. Species identification and virulence attributes of Saccharomyces boulardii. J Clin Microbiol 1998; 36: 2613-7.

[23] De Llanos R, Fernández-Espinar MT, Querol A. A comparison of clinical and food Saccharomyces cerevisiae isolates on the basis of potential virulence factors. Antonie Van Leeuwhenhoek 2006; 90: 221-31.

[24] Holzschu DL, Chandler FW, Ajello L, Ahern DG. Evaluation of industrial yeast for pathogenicity. Sabouradia 1979; 17: 71-8.

[25] Maejima K, Shimoda K, Morita C, et al. Colonization and pathogenicity of Saccharomyces cerevisiae MC16 in mice and cynomolgus monkeys after oral and intravenous administration. Jpn J Med Sci Biol 1980; 33: 271-6.

[26] Clemons KV, McCusker JH, Davis RW, Stevens DA. Comparative pathogenesis of clinical and nonclinical isolates of Saccharomyces cerevisiae. J Infect Dis 1994; 169: 859-67.

[27] Byron JK, Clemons KV, McCusker JH, et al. Pathogenicity of Saccharomyces cerevisiae in complement factor five-deficient mice. Infect Immun 1995; 63: 478-85.

[28] Okawa Y, Yamada Y. Lethality of yeast with low pathogenicity in mice immunocopromised by cyclophosphamide treatment. Biol Pharm Bull 2002; 25: 940-2.

[29] Wheeler RT, Kupiec M, Magnelli P, et al. A Saccharomyces cerevisiae mutant with increased virulence. Proc Natl Acad Sci USA 2002; 100: 2766-70.

[30] Klingberg TD, Lesnik U, Arneborg N, et al. Comparison of Saccharomyces cerevisiae strains of clinical and nonclinical origin by molecular typing and determination of putative virulence traits. FEMS Yeast Res 2008; 8: 631-40.

[31] Romani L. Immunity to fungal infections. Nat Rev Immunol 2004; 4: 1-23.

[32] Gozalbo D, Roig P, Villamón E, Gil ML. Candida and candidiasis: the cell wall as a potential molecular target for antifungal therapy. Curr Drug Targets Infect Disord 2004; 4: 117-35.

[33] Underhill DM. Macrophage recognition of zymosan particles. J Endotoxin Res 2003; 9: 176-80.

[34] Gantner BN, Simmons RM, Canavera SJ, et al. Collaborative induction of inflammatory response by dectin-1 and Toll-like receptor 2. J Exp Med 2003; 197: 1107-17.

[35] Villamón E, Gozalbo D, Roig P, et al. Toll-like receptor 2 is essential in murine defenses against Candida albicans infections. Microb Infect 2004; 6: 1-7.

[36] Gil ML, Gozalbo D. TLR2, but not TLR4, triggers cytokine production by murine cells in response to Candida albicans yeasts and hyphae. Microb Infect 2006; 8: 2229-34.

[37] Goodridge HS, Simmons RM, Underhill DM. Dectin-1 stimulation by Candida albicans or zymosan triggers NFAT activation in macrophages and dendritic cells. J Immunol 2007; 178: 3107-15.

[38] De Bernardis F, Adriani FD, Lorenzini R, et al. Filamentous growth and elevated vaginopathic potential of a non-germinative variant of Candida albicans expressing low virulence in systemic infection. Infect Immun 1993; 61: 1500-8.

[39] Villamón E, Gozalbo D, Roig P, et al. Myeloid differentiation factor 88 (MyD88) is required for murine resistance to Candida albicans and is critically involved in Candida-induced production of cytokines. Eur Cytokine Netw 2004, 15: 263-71.

[40] Villamón E, Gozalbo D, Roig P, et al. Toll-like receptor 2 is dispensable for acquired host immune resistance to Candida albicans in a murine model of disseminated candidiasis. Microb Infect 2004; 6: 542-8.

[41] Murciano C, Villamón E, Yáñez A, et al. Impaired immune response to Candida albicans in aged mice. J Med Microbiol 2006; 55: 1649-56.

[42] Murciano C, Yáñez A, Gil ML, Gozalbo D. Both viable and killed Candida albicans cells induce in vitro production of TNF- $\alpha$ and 
IFN- $\gamma$ in murine cells through a TLR2-dependent signalling. Eur Cytokine Netw 2007; 18: 1-5.

[43] Murciano C, Villamón E, O'Connor JE, et al. Killed Candida albicans yeasts and hyphae inhibit gamma interferon release by murine natural killer cells. Infect Immun 2006; 74: 1403-6.

[44] Murciano C, Yáñez A, O’Connor JE, et al. Influence of aging on murine neutrophil and macrophage function against Candida albicans. FEMS Immunol Med Microbiol 2008; 53: 214-21.

[45] Netea MG, Brown GD, Kullberg BJ, Gow NA. An integrated model of the recognition of Candida albicans by the innate immune system. Nat Rev Microbiol 2008; 6: 67-78.
[46] Murphy AR, Kavanagh KA. Adherence of clinical isolates of Saccharomyces cerevisiae to buccal epithelial cells. Med Mycol 2001; 39: 123-7.

[47] Klis FM, Mol P, Hellingwerf K, Brul S. Dynamics of cell wall structure in Saccharomyces cerevisiae. FEMS Microbiol Rev 2002; 26: $239-56$

[48] Klis FM, de Groot P, Hellingwerf K. Molecular organization of the cell wall of Candida albicans. Med Mycol 2001; 39 (Suppl 1): 1-8.

[49] Ruiz-Herrera J, Elorza MV, Valentin E, Sentandreu R. Molecular organization of the cell wall of Candida albicans and its relation to pathogenicity. FEMS Yeast Res 2006; 6: 14-29.

Received: January 9, 2009

Revised: March 25, 2009

Accepted: April 9, 2009

(C) Yáñez et al.; Licensee Bentham Open.

This is an open access article licensed under the terms of the Creative Commons Attribution Non-Commercial License (http://creativecommons.org/licenses/by$\mathrm{nc} / 3.0 /$ ) which permits unrestricted, non-commercial use, distribution and reproduction in any medium, provided the work is properly cited. 